\title{
Clinicopathological case: Rapid Cognitive Decline in an older
} man.

Shona Scott ${ }^{1}$, Jeremy Chataway ${ }^{2}$, Jon Stone ${ }^{1}$, Colin Smith ${ }^{3}$ and Richard Davenport ${ }^{1}$.

${ }^{1}$ Department of Clinical Neurosciences, Western General Hospital, Edinburgh, UK

${ }^{2}$ National Hospital for Neurology and Neurosurgery, Queen Square, University College Foundation NHS Trust, London

${ }^{3}$ Department of Neuropathology, Western General Hospital, Edinburgh UK

Correspondence to Dr Shona Scott : Neurology Registrar, Department of Clinical Neurosciences, Western General Hospital, Edinburgh, EH4 2XU UK: Shonamscott@nhs.net

This case was presented at the $36^{\text {th }}$ Edinburgh Advanced Neurology Course 2014

Key words:

Rapid cognitive decline, coma, dementia

Word count: 4098 


\section{Abstract}

A previously well older man died three weeks after presenting with confusion and hallucinations. $\mathrm{Dr}$ Chataway discusses the differential diagnosis of rapidly progressive cognitive symptoms, based on his presentation at the $36^{\text {th }}$ Edinburgh Advanced Neurology Course 2014.

\section{History}

An 83 year old man presented as an emergency with rapidly emerging confusion and visual hallucinations. His family had noted mild cognitive symptoms for a year or two, but he was living independently, and had recently enjoyed a family celebration.

He was in good health and his past medical history included angina and a cholecystectomy and transurethral prostatectomy. Eight years previously, his GP had noted an isolated episode when he awoke confused, making "out of context" comments about money to his wife. A CT head was reported as mildly atrophic for age, and he was referred to a psychogeriatrician, but he was never assessed as he recovered fully after this isolated episode. He was on no regular medication at the time of this admission.

On assessment his Abbreviated Mental Test (AMT) was 9/10 with a normal neurological examination. The following day he appeared more confused and agitated, and was treated with lorazepam and haloperidol. Two days later he was transferred for rehabilitation, with a diagnosis of delirium, possibly due to infection (see Table 1 for a summary of investigations).

On the rehabilitation ward, he was recorded to be "drowsy and disorientated in time and place". He had two falls over the next few days, but no new symptoms or signs were noted. On the fifth day of his admission, he became hypotensive and atrial fibrillation with a rapid ventricular rate was identified. A neurologist saw him the following day, and noted dysarthria with slurred, nasal speech, but no other focal signs. His conscious level and degree of confusion fluctuated, and on day 9 some myoclonic jerks were noted. Later that day he was transferred to the regional Neurosciences unit for further assessment, including MR head. Imaging was postponed however, as his airway was unsafe and soon after transfer, his airway safety deteriorated further, and he was intubated and ventilated (it was reasoned that although his prognosis was probably poor, there was no diagnosis). Further investigations were undertaken. He was given a single course of high dose intravenous methylprednisolone, without effect. After discussion with the family, it was decided to extubate him, and he died 24 hours later, on day 20 after admission to hospital. A limited post mortem was performed.

\begin{tabular}{|c|c|c|}
\hline \multicolumn{3}{|l|}{ Admission investigations } \\
\hline $\begin{array}{l}\text { - Chest Xray : normal } \\
\text { CT head (day 2): within } \\
\text { normal limits for age, } \\
\text { no evidence of } \\
\text { significant small vessel } \\
\text { disease. }\end{array}$ & $\begin{array}{l}\text { FBC, ESR, clotting screen, } \\
\text { electrolytes, creatinine, liver } \\
\text { and thyroid function tests, } \\
\text { calcium, albumin, creatine } \\
\text { kinase - normal } \\
\text { - Urea } 10.8 \mathrm{mmol} / \mathrm{L}(2.5-6.6)\end{array}$ & - Urine culture: normal \\
\hline \multicolumn{3}{|l|}{ Days 5 - 8 of illness } \\
\hline $\begin{array}{l}\text { Chest Xray bilateral } \\
\text { basal consolidation } \\
\text { with progression } \\
\text { between day } 5 \text { and } 8 \text {. } \\
\text { - CT head (day 6, after }\end{array}$ & $\begin{array}{l}\text { - WCC remained normal but } \\
\text { CRP rising. } \\
\text { - Urea rising to } 18.8\end{array}$ & \\
\hline
\end{tabular}




\begin{tabular}{|c|c|c|}
\hline $\begin{array}{l}\text { fall): within normal } \\
\text { limits for age. }\end{array}$ & & \\
\hline \multicolumn{3}{|l|}{ Days 9-21 in ICU } \\
\hline $\begin{array}{l}\text { MR head (day 15): } \\
\text { bilateral } 10 \mathrm{~mm} \\
\text { subdural hygromas, } \\
\text { new in appearance } \\
\text { compared to CT. No } \\
\text { other abnormalities }\end{array}$ & $\begin{array}{l}\text { - Acetyl choline receptor, Anti } \\
\text { MuSK, thyroid peroxidase, } \\
\text { neuronal, voltage gated } \\
\text { potassium channel and NMDA } \\
\text { receptor antibodies negative } \\
\text { - Auto-immune screen: normal } \\
\text { - } \text { CRP (day } 10): 56 \mathrm{mg} / \mathrm{L}(\leq 5)\end{array}$ & $\begin{array}{l}\text { CSF examination (day } \\
\text { 10): normal } \\
\text { EEG (day 9): poorly } \\
\text { tolerated: generalised } \\
\text { slowing but no focal } \\
\text { or epileptic features }\end{array}$ \\
\hline
\end{tabular}

Table 1: Summary of investigations

\section{Discussion by Dr Jeremy Chataway}

I was hoping for a white matter case, perhaps MELAS, vanishing white matter disease or leucodystrophies ${ }^{1}$ - but it's not to be! This is about an 83-year-old gentleman who presented to A\&E and was a bit confused, had a normal CT head scan, then died within three weeks. He was well in the period leading up to admission.

So I thought I would introduce a Sherlock Holmes theme because Conan Doyle was of course born in Edinburgh and attended Edinburgh Medical School. He began writing when no patients came to his practice and eventually after several moves set up an Ophthalmology practice near Baker Street but again nobody came so he kept writing. The rest is history and so you will see an astute almost Holmesian approach to this CPC!

Gregory (Scotland Yard detective); "Is there any point to which you wish to draw my attention?" Sherlock Holmes; "The curious incident of the dog in the night-time"

Gregory; "The dog did nothing in the night-time"

Sherlock Holmes; "That was the curious incident".

The Adventures of Silver Blaze. Sir Arthur Conan Doyle 1893

"So eliminate all other factors and the one that remains must be the truth"

A Study in Scarlett. Sir Arthur Conan Doyle 1897

And there are undeniable similarities between Professor Moriaty and Dr Richard Davenport, the organiser of this course. 'I'm going to break you Holmes, I'm going to bring off right under your nose the most incredible crime of the century, and you'll never suspect it until it's too late. That will be the end of you Mr. Sherlock Holmes. And when I've beaten and ruined you then I can retire in peace. I'd like to retire; crime no longer amuses me. I'd like to devote my remaining years to abstract science'. However, we must proceed with the case. 
His deterioration was rapid with visual hallucinations on a background of mild cognitive symptoms for a couple of years. He had fairly standard general medical complaints: heart disease, prostate problems and GI disturbance and he was not on any medication.

\section{Mild cognitive impairment ( $\mathrm{MCl})$}

First we will explore $\mathrm{MCl}$ and why and how that could that be important. $\mathrm{MCl}$ is a function of age, but it can be on a spectrum that may progress to Alzheimer's dementia (AD). It is defined as a memory complaint when other aspects of cognitive function are within normal limits and activities of daily living are maintained. In patients with $\mathrm{MCl}$ the rate of development of $A D$ is about $12 \%$ per year compared to about $2 \%$ per year in controls without $\mathrm{MCl}^{2}$.

\section{Visual hallucinations}

There are a number of conditions that cause visual hallucinations so maybe the answer lies here. Table 2 summarises some of the terms used to describe visual hallucinations/distortions and lists conditions that may cause visual hallucinations.

\begin{tabular}{|l|l|}
\hline Term & Feature \\
\hline Tesselopsia/teichopsia & Regularly repeating patterns \\
\hline Dendropsia & Branching patterns \\
\hline Hyperchromatopsia & Perception of exceptionally wild and brilliant colours \\
\hline Polyopia & Seeing a single target as multiple images \\
\hline Entomopia & $\begin{array}{l}\text { Rare form of polyopia when images seen as multiple rows } \\
\text { and columns (insect vision) }\end{array}$ \\
\hline Macro/micropsia & $\begin{array}{l}\text { Images perceived as bigger or smaller than they actually } \\
\text { are }\end{array}$ \\
\hline Metomorphosia & Distorted images \\
\hline Palinopsia & $\begin{array}{l}\text { Image persists in visual field after stimulus has left the } \\
\text { visual field }\end{array}$ \\
\hline $\begin{array}{l}\text { Visual allesthesia } \\
\text { Transportation of an object from one visual field to } \\
\text { another }\end{array}$ \\
\hline Prosopometamorphosia & Illusion of distorted faces \\
\hline Oscillopsia & Objects in the visual field appear to oscillate \\
\hline $\begin{array}{l}\text { Conditions associated with visual hallucinations: maybe the answer lies here. } \\
\text { Delirium and delirium tremens, drugs and toxins, Dementia with Lewy Bodies and other } \\
\text { parkinsonian syndromes, Alzheimer's disease, Posterior Cortical Atrophy, epilepsy, } \\
\text { sleep disorders and narcolepsy, migraine, Huntington's disease, structural brain lesions, } \\
\text { stroke, Prion diseases especially the Heidenhain CJD variant, Niemann-Pick disease, } \\
\text { encephalitis, inborn errors of metabolism, visual impairment (Charles Bonnet } \\
\text { syndrome) }\end{array}$ \\
\hline
\end{tabular}

Table 2: Different types of visual hallucination/disturbance and conditions that may cause visual hallucinations ${ }^{3}$

\section{Further analysis}

Initially he had a normal neurological examination and an AMT 9/10, but became confused and agitated requiring lorazepam and haloperidol. A few days later he was hypotensive with deranged electrolytes and $\mathrm{AF}$, then became comatose requiring intubation. Was this neurological disease or 
evidence of a more systemic illness? The only neurological sounding symptom was nasal speech but no other signs were identified. Myoclonic jerks were noted (day 9) raising the possibility of prion disease, but it could also be metabolic derangement or infection.

\section{Coma}

Consciousness requires arousal and awareness and depends on a functioning ascending reticular activating system and the cerebral cortex ${ }^{4}$. Table 3 provides the features that distinguish encephalopathy and encephalitis. In addition to structural causes of coma there are many others including metabolic derangement, seizures and infection.

Encephalopathy

- Symptoms persisting at least 24 hours

- Depressed or altered level of consciousness including:

○ Lethargy or

- Extreme irritability or

- Significant change in personality or behaviour and

- no other cause found

\section{Encephalitis (infectious or autoimmune)}

- Encephalopathy + two or more of

- pyrexia $>37.5^{\circ} \mathrm{C}$

- seizure(s)

- focal neurological findings

- CSF pleocytosis ( $>5 \mathrm{WBC} /$ microliter)

$\circ$ characteristic abnormal findings on neuroimaging (CT or MRI)

Table 3: Criteria for diagnosing encephalopathy and encephalitis ${ }^{5}$

\section{Identifying the cause of coma}

Once general medical causes of coma have been excluded then primary neurological causes should be considered, either central or peripheral. Patients might have both neurological and nonneurological causes either simultaneously or sequentially. In addition, there are important causes of encephalopathy not to overlook:

1. Wernicke's encephalopathy (triad of ophthalmoplegia, encephalopathy and gait ataxia): alcohol consumption may be denied or hyperemesis of pregnancy overlooked. The lesions of chronic Wernicke's occur in a characteristic, symmetrical distribution in structures surrounding the aqueduct and the third and fourth ventricles. It typically causes atrophy of the mammillary bodies and dorsomedial thalami. Always remember that dextrose prior to thiamine replacement may precipitate Wernicke's. Confusion is the most common symptom of Wernicke's, followed by staggering gait and ocular problems but lethargy or coma may be the only symptom.

2. Drugs; prescription drugs e.g. neuroleptic malignant syndrome, as well as illicit drugs.

3. Trauma; isodense or hidden subdurals ${ }^{6}$.

4. Common systemic infection can cause coma but rarer infections such as endocarditis or HIV can present as coma. Be alert to relevant infectious causes in the returning traveller (e.g. malaria).

5. Respiratory and metabolic derangements include: pulmonary causes (Obstructive Sleep Apnoea, hypercapnia, hypoxia and carbon monoxide poisoning); renal (uraemia and haemolytic uraemic syndrome); hepatic (acute liver failure or hepatic encephalopathy); haematological (thrombotic thrombocytopenic purpura, lupus anticoagulant syndrome); and endocrine (diabetic ketoacidosis or hypoglycaemia, hypothyroidism, hypercalcaemia, hyponatraemia, Hashimoto's encephalopathy). 
More rare causes of coma, easily forgotten, include Addison's disease (when the sodium may not be that low), insulinoma, phaeochromocytoma and non-CNS malignancy. Pseudo-coma due to psychogenic causes may occasionally catch out even the most experienced.

In this case no medical cause was found: blood tests were normal, the chest $x$-ray showed signs of pneumonia but only as his condition deteriorated, and urine was sterile. Therefore, it is likely to be a primary neurological cause of coma. A clue may be the bilateral subdural hygromas seen on MR, not evident on CT. However we would normally discount hygromas as a cause of pathology, although they are occasionally seen in conditions such as CNS lymphoma. In this case I conclude that the imaging is unremarkable, and not offering diagnostic clues. As well as unremarkable imaging, the CSF and other blood tests were all within normal limits.

"Stay with me here. Sherlock says it is a capital mistake to theorize before you have all the evidence. It biases the judgement"

\section{Rapidly progressive dementias}

Delirium should be considered in all patients presenting with acute fluctuating mental states such as disturbed attention, disorganised thinking and altered level of consciousness. Rapidly progressive dementias (RPD) are defined as dementias that evolve over days to weeks or sub acutely over months ${ }^{7}$. Analysis from the five largest case series of RPD identified 675 patients, with a cause established in $95 \%$ of cases. Over half of the cases were accounted for by CJD (58\%), neurodegenerative conditions (e.g. AD 22\%), with up to $19 \%$ being attributed to treatable causes (Wernicke's, B12 deficiency and toxic/metabolic deficiencies) ${ }^{7}$.

Our patient was noted to have visual hallucinations, which may suggest a neurodegenerative condition. Table 4 summarises the neurodegenerative conditions that cause RPD but most of these are non-starters here as the duration from $\mathrm{MCl}$ to coma and death was so short. $A D$ is unlikely to be this rapid unless his decline had been begun earlier than his family realised, nor would it fit with MND or Corticobasal Degeneration, but Prion disease is possible (and of course we are at the home of Prion disease). CJD can be extremely quick and deterioration to death within 25 days has been reported but a more typical duration would be 6 to 18 months $^{7}$. Ante-mortem diagnosis of Prion disease can be difficult and is based on: MRI (classically showing cortical ribboning, basal ganglia and thalamus hyperintensity on diffusion weighted imaging and fluid attenuated inversion recovery), EEG (generalised slowing and periodic polyspike-wave complexes and sharp waves which may not become apparent until late in the disease) and CSF analysis (normal or minimally raised WCC, total tau protein elevated to $>1200$ picograms $/ \mathrm{mL}$, protein 14.3.3 positive on Western blot. S100b may have a role in supporting a diagnosis if the clinical suspicion of CJD is high. RT-QulC is currently being developed as an accurate pre-mortem diagnostic test).

\begin{tabular}{|ll|}
\hline $\begin{array}{l}\text { Neurodegenerative } \\
\text { conditions }\end{array}$ & Alzheimer's disease (AD) \\
& Corticobasal degeneration (CBD) \\
& Dementia with Lewy bodies (DLB) \\
& Familial spastic paraparesis \\
& Frontotemporal degeneration (FTD) \\
& Motor neurone disease (MND) \\
& Progressive supranuclear palsy (PSP) \\
& Progressive subcortical gliosis \\
\hline
\end{tabular}




\begin{tabular}{|ll|}
\hline & Prion disease \\
\hline Autoimmune or & Acute disseminated encephalomyelitis(ADEM) \\
conditions & Anti-body mediated brain disease \\
& Anti-GAD65 autoimmunity \\
& Behcet's disease \\
& Coeliac disease \\
& Limbic encephalitis \\
& Multiple sclerosis (MS) \\
& Sjogren's syndrome \\
& Steroid responsive encephalopathy \\
& Cerebral autosomal-dominant arteriopathy with sub \\
& acute infarcts and leukoencephlopathy (CADASIL) \\
& Cerebral amyloid angioathy \\
& CNS vasculitis \\
Vascular & Mitochondrial Encephalopathy with lactic acidosis and \\
& stroke like symptoms (MELAS) \\
& Strategic infarction \\
& Subdural haematoma \\
& Vascular dementia \\
\hline Cerebrotendinous xanthomatosis \\
Extrapontine mylinosis \\
Liver failure \\
MELAS \\
Neurodegeneration with brain iron accumulation (NBIA) \\
Neuronal ceroid lipofuscinosis \\
Nutritional deficiency (B1, B3, B12 or folate) \\
Porphyria \\
Uraemia \\
\hline Metabolic disorders
\end{tabular}

Table 4: causes of rapidly progressive dementias ${ }^{7}$

\section{CNS Infections}

Could this be infection? A review of admissions to a neurological intensive care found the most common primary cause was infection ${ }^{8}$. Herpes simplex virus (HSV) accounts for about $10 \%$ of the viral encephalitides ${ }^{8}$. Negative investigations for HSV do not completely exclude the diagnosis but should be considered a 'red flag' to prompt further investigation for other infectious causes such as VZV, EBV, CMV, measles, rubella, aborvirus, enterovirus, TB, fungal, parasitic (Toxoplasma and malaria), Rickettsia, Listeria and cryptoccocus. Non-infectious causes should also be considered including CJD, paraneoplastic, and inflammatory conditions such as Behçet's. The normal CSF and brain imaging count against an infectious cause and, on balance I am discounting an infectious cause.

\section{Vascular}

Vascular causes such as stroke, venous sinus thrombosis and sub arachnoid haemorrhage are unlikely particularly in the context of normal imaging and progressive symptoms, but vasculitis is a background contender. However, I would expect to see some abnormalities on the brain imaging and perhaps CSF.

\section{Paraneoplastic and neoplastic syndromes}


Another consideration would be a paraneoplastic syndrome ${ }^{9}$ typically associated with small cell lung cancer, breast cancer or germ cell tumours, and of course paraneoplastic syndromes can precede detectable tumours by months, sometimes years. Central paraneoplastic syndromes, such as cerebellar degeneration, are often associated with lung cancer. Peripheral paraneoplastic syndromes such as LEMS also occur. A central paraneoplastic brainstem encephalomyelitis would be high on my differential diagnosis and although anti neuronal antibodies were negative; it may be one that has not been tested. However, we would normally expect the brain imaging to show abnormalities, particularly around the medial temporal lobes, but this is not always the case. The entirely normal CSF would be against a diagnosis of paraneoplastic encephalitis as we normally expect a lymphocytosis, oligoclonal bands or a raised protein. Primary CNS malignancy such as lymphoma should also be considered.

\section{Autoimmune disease}

A further thought would be autoimmune encephalitis ${ }^{10}$ as the patient was confused and rapidly deteriorated to coma with few localising signs. However the autoantibody screen was negative and therefore this is unlikely, but again it could be an autoantibody that has not been tested. Imaging can be normal in about $40 \%$ of autoimmune encephalitis so don't discount it as a diagnosis solely on normal imaging findings.

As Sherlock would say in "The Sign of Four" (1890):

"What seems strange to you is only so because you do not follow my train of thought or observe the facts upon which large inferences may depend."

\section{Peripheral nervous system causes including the neuromuscular junction}

Disorders of the peripheral nervous system such as acute polyneuropathies, or of the neuromuscular junction such as myasthenia gravis, or drugs and toxins can lead to coma. The absence of focal signs on examination including the normal reflexes strongly point away from peripheral causes including Acute Inflammatory Demyelinating Polyradiculopathy (AIDP). However, the team did checked antiacetylcholine receptor and MuSK antibodies, which would suggest they had suspicions of a peripheral cause. On balance the lack of signs would argue against the peripheral nervous system as the primary site of pathology. This case therefore must have a central nervous system cause.

\section{Cardiac and respiratory diagnoses}

Were his new cardiac signs (AF) a clue to the diagnosis? We know of neurological causes of cardiac signs following subarachnoid haemorrhage and ischaemic stroke. Could endocarditis be the cause all along? Probably not and I think AF was more of a reflection that he was old and ill with pneumonia.

As Sherlock would say:

"Eliminate the impossible, whatever remains however improbable must be the truth."

So two likely candidates remain: 
1. Paraneoplastic - the three-week time course to death would be possible however the negative antineuronal screen and the normal CSF would count against this diagnosis.

2. Prion disease: sporadic CJD - but the speed seems a little quick although if we go back to the history it may be longer as he had an episode of nocturnal confusion and some cognitive change.

\section{Jeremy Chataway's diagnosis}

Prion Disease - a home from home. "There is nothing new under the sun: it's all been done before".

\section{Questions from the audience}

Q: How much alcohol did he consume?

RD: His alcohol history was modest

Q: Did you consider Lewy body as it can have an aggressive course?

JC: Normally you would expect it to be initially reversible rather than this relentless aggressive decline into coma. He was given an antipsychotic and it's possible that accelerated his decline.

Q: Would you expect to see fluctuations in prion disease?

JC: No, but the fluctuations could be explained by superadded infection such as pneumonia.

Q: Would you expect to see some signs in prion disease?

JC: He was drowsy and dysarthric and by the time he was ventilated it would be difficult to identify signs or progression of signs. Myoclonus was noted.

Q: What was the nature of the visual hallucinations?

JS: He was wandering in a confused, aggressive and agitated state, and his hallucinosis appeared to involve people and objects. This occurred within 12 hours of admission and was severe enough that the admitting team treated him with haloperidol and lorazepam.

Q: Was there a history of sleep disturbance prior to admission?

RD: This was not specifically asked about - I assume you are wondering about REM behavioural disturbance as the harbinger of an alpha-synucleinopathy?

Q: Is it possible for this to be autoimmune encephalitis with normal imaging and CSF?

RD: Yes, all things are possible, although as Dr Chataway has identified, it would be unusual with all the tests being normal, as in this case.

\section{Audience diagnoses}

Half the audience agreed with a Prion disease diagnosis, paraneoplasia found much less favour. 
JS: A final comment prior to the denouement: the family were keen for the case to be presented and discussed at this CPC as a learning point. They felt haloperidol was the cause of his rapid decline and were keen for a pathological diagnosis. We are grateful them for allowing us to present this CPC.

\section{Pathology review by Dr Colin Smith}

Consent for a limited post mortem was obtained. Macroscopically the brain was a normal weight with mild symmetrical frontotemporal atrophy evident. Microscopic examination of a number of brain regions using standard haematoxylin and eosin (H\&E) stains identified significant cerebrovascular disease affecting the large leptomeningeal vessels with prominent, widespread atherosclerosis but no acute thrombus. In addition, small vessel arteriolosclerosis was noted in a number of regions with some white matter rarefaction but no lacunar infarcts.

Neuritic plaques and neurofibrillary tangles were obvious in the entorhinal cortex on H\&E stain, and highlighted by $\beta$-amyloid and tau immunohistochemistry. There was no evidence of spongiform encephalopathy, but Lewy bodies were seen in the substantia nigra, with clear loss of pigmented neurones and extra neuronal pigment. $\alpha$-synuclein staining highlighted neuronal inclusions within the substantia nigra, along with Lewy neurites (abnormal parenchymal threads) (figure 1). Striking and typical $\alpha$-synuclein pathology was also seen in the dorsal vagal nucleus (figure 2 ), and cortical Lewy bodies were noted (figure 3 ). In the occipital region there was evidence of cerebral amyloid angiopathy, but this was not seen in other brain areas. This was mainly in the leptomeningeal and only occasionally the parenchymal vessels.

In terms of staging of the Lewy body disease, which can be done using the either the Braak ${ }^{11}$ or consortium guidelines ${ }^{12}$, this is at least Braak stage 5 as the cingulate gyrus was involved and this would equate to the neocortical stage of Lewy body disease using consortium guidelines. For Alzheimer's disease associated pathology we can also use Braak staging for the neurofibrillary pathology and more recent guidelines also bring in amyloid with comments on Lewy body pathology. The AD pathology here is Braak stage I-II.

\section{Main pathological findings}

The final pathological diagnosis is therefore: diffuse Lewy Body Disease Braak stage 5; Alzheimer's pathology Braak stage I- II; and cerebrovascular disease in the form of large vessel atherosclerosis, small vessel arteriolosclerosis and occipital cerebral amyloid angiopathy. This is on a background of pneumonia and hypoxaemia causing brain hypoperfusion and led to the more widespread changes seen.

\section{Comments}

JC: Was it the combination of antipsychotics and systemic infection that resulted in his rapid decline?

JS: A background of cerebrovascular disease and infection may explain the explosive onset and both are likely to have accelerated the process. Exposure to antipsychotics may have played a part as well, although he did not develop the typical dramatic parkinsonism that is often the consequence of antipsychotic use in DLB.

Q: So clinically was this Dementia with Lewy Bodies (DLB) or AD? 
CS: We frequently see Braak stage 1-2 Alzheimer's pathology in patients who were said to be cognitively normal. However we never see Braak stage 5 LBD in patients without clinical signs of LBD. The aim of the McKeith consortium approach was to help answer this question with regard to the relative importance of either pathology as a cause of death. This was a neocortical case and therefore the predominant pathology.

RD: Clinically we can retrospectively acknowledge the rapid onset and visual hallucinosis as consistent with a diagnosis of DLB, and this is now supported by the pathology.

Q: What are we attributing the myoclonus to?

RD: It is not an uncommon finding in end stage cognitive disease.

Q: Had he died of, say, a heart attack one month prior to this presentation, would the brain pathology have been the same?

CS: Yes, the clinical picture was very rapid but the pathology would have been present before his decline.

\section{Final Clinico-Pathological Diagnosis}

Dementia with Lewy Bodies

\section{Dementia with Lewy Bodies.}

Lewy bodies are named after Frederick Lewy who in 1912 described abnormal intracytoplasm inclusions in a patient with Parkinson's disease; cortical Lewy bodies were finally linked with a dementia in $1961^{13}$. Currently it is estimated that dementia affects $7 \%$ of people over the age of 65 years and $30 \%$ of those older than 80 years ${ }^{14}$. Dementia with Lewy bodies (DLB) is the second most common degenerative dementia accounting for $10-20 \%$ of cases at post-mortem ${ }^{14,15}$. If a patient has a progressive cognitive decline within a year of onset of motor parkinsonism then this is considered to be $\mathrm{DLB}^{13}$, although it is likely that DLB and PD associated dementia form a continuum.

The main differential diagnosis in cases of DLB is AD, PD associated dementia, atypical PD syndromes such as progressive supra nuclear palsy or cortico-basal degeneration and Creutzfeldt-Jakob disease $^{13}$. In PD the distribution of Lewy bodies is subcortical and the progression of Lewy body pathology is usually in a caudal to rostral direction i.e. from nigra to limbic regions and finally to the neocorte ${ }^{16}$. In contrast a more generalised distribution is seen in patients with DLB ${ }^{14}$. Levo-dopa response is often poor in patients with DLB, and they can be acutely sensitive to even small doses of neuroleptic drugs ${ }^{17}$.

\section{Lewy body Pathology}

Lewy bodies are inclusion bodies containing mainly $\alpha$-synuclein and are probably markers of neuronal distress. They are common in the ageing brain and are associated with neurodegenerative conditions ${ }^{16}$, but the role of Lewy bodies in disease is not straightforward. A longitudinal community based clinical-pathological study of 872 subjects found 157 subjects with Lewy bodies in the substantia nigra, limbic and neocortical regions. Of these 157 patients, 103 (66\%) had a pathological diagnosis of AD but only half had detectable dementia at death and one third of people without 
Lewy body pathology had shown signs of dementia prior to death. However, Lewy body pathology of the neocortical type was associated with increased odds of dementia and a more rapid decline in all cognitive domains, and limbic-type Lewy body pathology was associated with poor visuospatial skills ${ }^{16}$. Not all patients with pathologically defined DLB are clinically demented.

DLB should be suspected in older people with mild memory impairment and visual hallucinations but an early feature may be recurrent periods of confusion ${ }^{13,14,17}$. Typically the episodic confusion is on a background of progressive cognitive deterioration with the fluctuations in cognition mainly associated with inattention and loss of alertness. Psychiatric manifestations occur early in the disease with visual hallucinations, delusions, apathy and anxiety being the most commonly reported ${ }^{13}$. Hallucinations are usually vivid, colourful, three-dimensional and are generally mute images of animate objects. They are recurrent and well formed, consisting of small animals, people or children. Misperceptions such as mistaking carpet and wallpaper patterns for snakes is common $^{3,13,14}$. Visual hallucinations are associated with lower cortical acetylcholine levels and consequently may respond well to cholinesterase inhibitors. Dizziness, pre-syncope, syncope and falls due to orthostatic hypotension or carotid sinus hypersensitivity are important other early symptoms that may support the clinical diagnosis. In addition, prominent REM behavioural sleep disorder may be reported ${ }^{16}$. A discussion of the full criteria for the diagnosis of DLB and the features that are useful in making a clinical diagnosis of DLB is available ${ }^{15}$.

\section{Rapidly progressive DLB}

Typically DLB has a progressive course with mean duration from symptom onset to death of 5-8 years ${ }^{18}$. However, case reports of rapid disease progression to death within 3-18 months of symptom onset can be found ${ }^{18}$. Diagnosis of DLB in patients with rapid cognitive decline can be extremely difficult because the neurological symptoms are non-specific and the investigations unremarkable. Delirium at symptom onset may be a marker of rapidly progressive DLB, but no biological substrate has been identified to predict which patients will have a rapidly progressive course.

A review of cases from a neurological tissue bank in Spain identified 83 pathologically confirmed cases of DLB (36 with a clinical diagnosis of PD with or without dementia and 47 with a clinical picture of DLB $)^{18}$. Of these 83 patients, six patients had a symptom onset to death of less than 18 months ( 4 male: 2 female, mean age onset 72.5 years). Of these six, two were noted to have auditory hallucinations, four had mild Parkinsonism and three recurrent falls. Three patients initially presented with an acute confusional state requiring emergency hospital admission. Two of these three patients had mild cognitive symptoms for several months prior to onset and all had wellformed visual hallucinations and structured delusions. Action and spontaneous myoclonus were reported in three patients and two patients had a significant deterioration after treatment with antipsychotics. Brain MRI scans revealed moderate atrophy but no cortical or basal ganglia abnormalities. The pathological findings were diffuse Lewy body in all patients with 4 cases with neocortical-diffuse pathology and 2 limbic type pathology. There were no atypical findings in terms of extension or severity of Lewy bodies or other pathology. Five of the cases had coexisting AD type pathology (Braak stage II - VI), four had mild amyloid angiopathy and one had small vessel pathology ${ }^{18}$.

\section{Neuroleptic Sensitivity}


Up to $60 \%$ of demented patients in hospital receive antipsychotic medications ${ }^{17}$. Patients with DLB may be particularly at risk of prolonged or permanent cognitive decline following surgery or exposure to antipsychotic mediation ${ }^{7}$. Severe neuroleptic sensitivity is characterised by sudden onset sedation, increased confusion, immobility and muscle rigidity and up to $30-50 \%$ of patients with DLB experience severe reactions even at low doses which can be fatal within days to weeks ${ }^{13,15}$.

Significant striatal $\alpha$-synuclein pathology and neuronal loss or dysfunction within the putamen may provide the pathophysiological reason for neuroleptic sensitivity in patients with DLB and related parkinsonism conditions ${ }^{15}$. A prospective study of patients exposed to antipsychotic medication included 15 patients with DLB; eight of the fifteen (53\%) patients experienced severe neuroleptic sensitivity. In another series of 20 demented patients with DLB who died in hospital, 80\% (16/20) received antipsychotic medication and of these $81 \%(13 / 16)$ showed severe sensitivity to these medications and in 7 (54\%), the reaction appeared to precipitate their terminal decline ${ }^{13}$. However, a history of neuroleptic tolerance is compatible with a diagnosis of DLB ${ }^{19}$.

\section{Conclusion}

Rapidly progressive dementias can be difficult diagnose as there may be few localising features and delirium is often suspected ${ }^{19}$. Families may not have appreciated the proceeding progressive mild cognitive impairment and initial cognitive fluctuations are discounted or forgotten about, as they are often short-lived with a seemingly good recovery. DLB typically has a slower course and may therefore be over-looked as a cause of RPD.

Contributors

SS took primary responsibility for writing this article and undertook a literature review. JC provided the critical appraisal of the case, CS provided the pathology and pathological discussion. JS and RD contributed towards writing the article.

\section{References}

1. Ahmed RM, Murphy E, Davagnanam I, et al. A practical approach to diagnosing adult onset leukodystrophies J Neurol Neurosurg Psychiatry 2014;85: 770-781.

2. Petersen RC, Smith G, Waring S, Ivnik R, Tangalos EG, Kokemen (1999) Mild cognitive impairment: clinical characterization and Outcome. Arch Neurol 56:303-308, 1999

3. FFytche D, Blom JD, Catani M (2010) Disoders of visual perception. J Neurol Neurosurg Psychiatry 81: $1280-7$.

4. Walters RJL, Wills A, Smith P (2007) Specialist training in Neurology. Elsevier, Edinburgh pp109

5. Venkatesan AR, Tunkel KC, Lauring AS, Sejvar J, Bitnun A, Stahl JP (2013) Case definitions, Diagnostic algorithms and Priorities in Encephalitis: Consensus Statement of the International Encephalitis Consortium. Clinical Infectious Diseases 57: 1114-28. 
6. Davenport RJ (1994) Lesson of the Week: Detection of bilateral isodense subdural haematomas BMJ 1994;309:792

7. Day GS \&Tang-Wai DF (2014) When dementia progresses quickly: a practical approach to diagnosis and management of rapidly progressive dementia. Neurodegenerative disease Management 2014 4(1) 41-56

8. Howard RS, Kullmann DM, Hirsch NP (2003) Admission to neurological intensive care: Who, when and why? J Neurol Neurosurg Psychiatry 74: suppl iii, iii2-9.

9. Dalmau M, Rosenfeild MR (2008) Paraneoplastic syndromes of the CNS. Lancet Neurology 7:327140

10. Vincent A Bien CG, Irani SR, Waters P (2011) Autoabtibodies associated with disease of the CNS: new developments and future challenges. Lancet Neurology 10: 759-72

11. Braak H et al. (2003) Staging of brain pathology related to sporadic Parkinson's disease. Neurobiol. Aging 24, 197-211

12.McKeith et al. Consensus guidelines for the clinical and pathologic diagnosis of dementia with Lewy bodies (DLB): report of the consortium on DLB international workshop. Neurology 47, 11131124 (1996)

13. Huang $Y$ and Halliday $G$ (2013) Can we clinically diagnose dementia with Lewy bodies yet? Translational Neurodegeneration 2:4

14. McKeith I, Mintzer J, Aarsland D et al (2004) Dementia with Lewy bodies. The Lancet Neurology. 3: $19-28$

15. Aarslan D, Perry R, Larsen JP, McKeith IG, O'Brien JT, Perry EK, Burn D, Ballard CG (2005) Neuroleptic sensitivity in Parkinson's disease and Parkinsonian dementias J Clin Psychiatry 66:633-7

16. Schneider JA, Arvanittakis Z, Yu L, Boyle PA, Leurgans SE, Bennett DA (2012) Cognitive impairment, decline and fluctuations in older community-dwelling subjects with Lewy bodies. Brain 135: 3005-14.

17. McKeith I, Fairbairn A, Perry R, Thompson P, Perry E (1992) Neuroleptic sensitivity in patients with senile dementia of Lewy body type. BMJ 305 (6855) 673-8

18. Gaig C, Valldeorioia, Gelpi E, Ezquerre M, Llufriu S, Buongiorno M. Rey M, Marti M, Graus F Tolosa E (2011) Rapidly progressive diffuse Lewy body disease. Movement disorders 26:7 1316-23

19.McKeith IG, Dickson DW, Lowe J et al (2005) Diagnosis and management of dementia with Lewy bodies: third report of the DLB consortium. Neurology 65: 1863-72.

List of figures

Figure 1a: $\alpha$-synuclein positive intra-neuronal inclusions and Lewy threads within the substantia nigra ( $\alpha$-synuclein immunohistochemistry $x 40$ ) 
Figure 2: Dorsal motor vagal nucleus $\alpha$-synuclein pathology, with both intra-neuronal inclusions and threads ( $\alpha$-synuclein immunohistochemistry x10)

Figure 3: Cortical Lewy bodies within the cingulate gyrus, highlighted with $\alpha$-synuclein immunohistochemistry ( $\alpha$-synuclein immunohistochemistry x20).

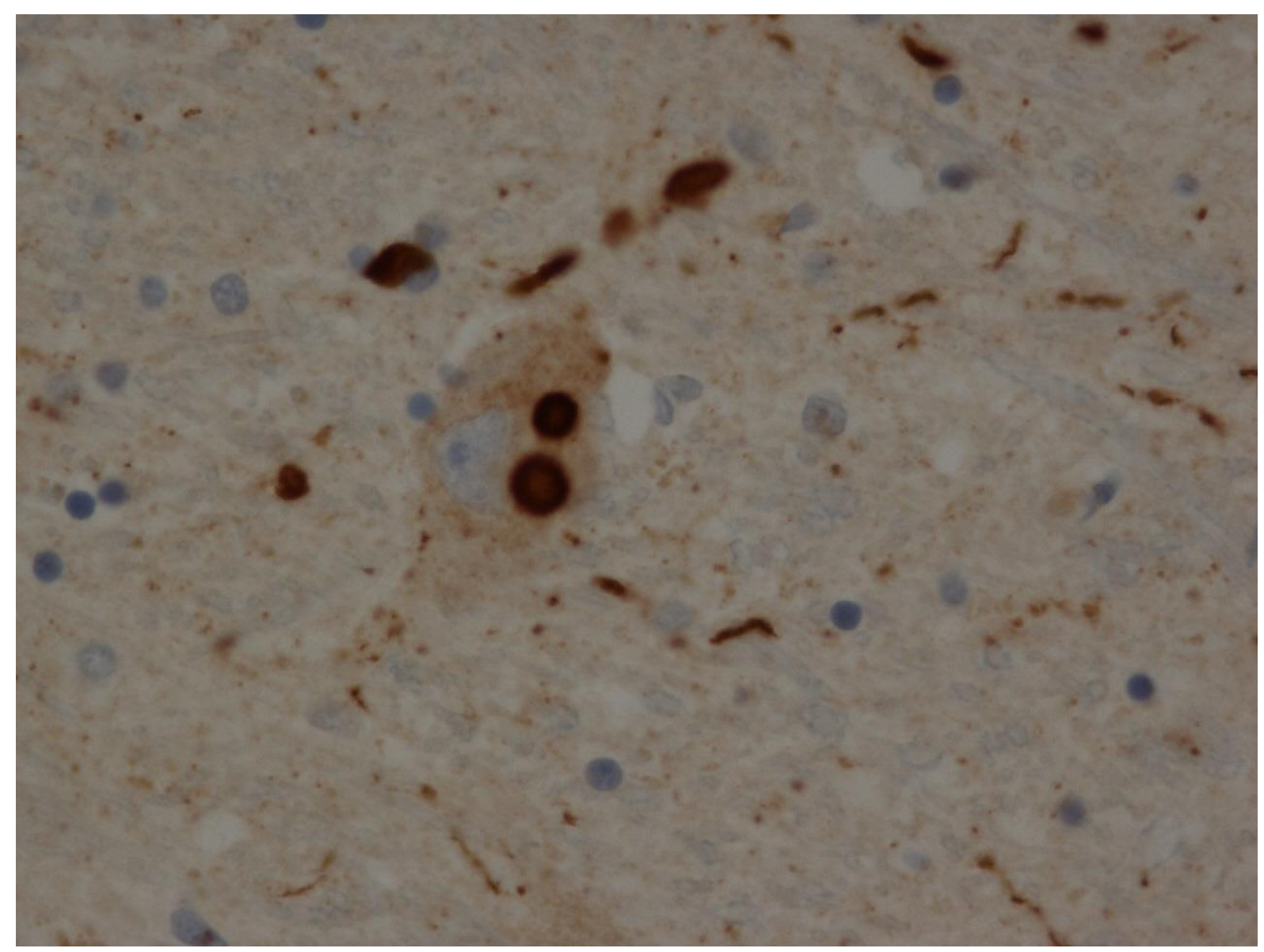





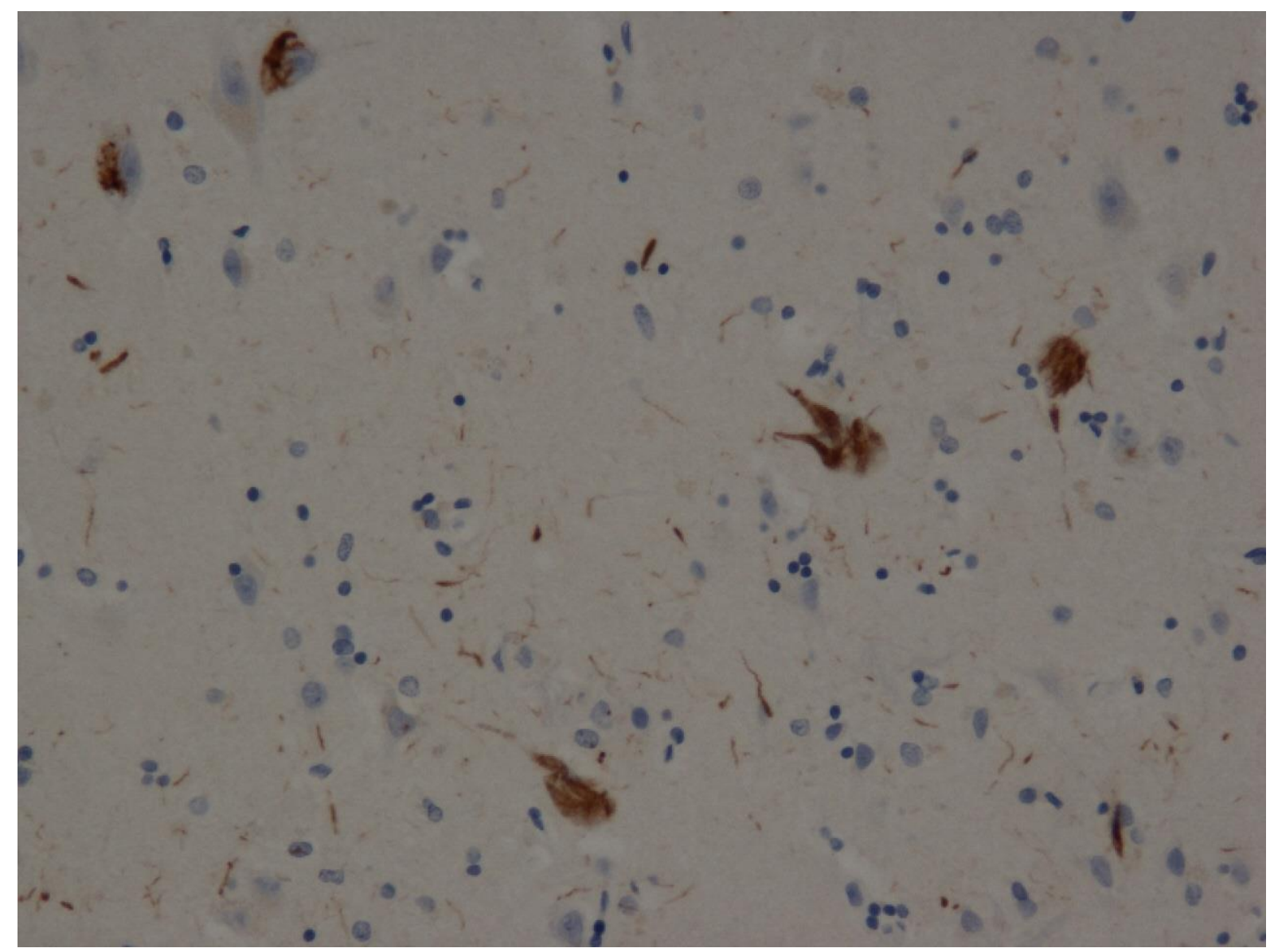

